\title{
Comparative Analysis of Features for Determining State of Health in Lithium-Ion Batteries
}

\author{
Nick Williard, Wei He, Michael Osterman, and Michael Pecht \\ Center for Advanced Life Cycle Engineering, College Park, Maryland, 20742, USA \\ nwilliar@calce.umd.edu \\ weihe@calce.umd.edu \\ osterman@calce.umd.edu \\ Pecht@calce.umd.edu
}

\begin{abstract}
Traditionally, capacity and resistance have been used as the features to determine the state of health of lithium-ion batteries. In the present study, two additional features, the length of time of the constant current and the constant voltage phases of charging were used as additional indicators of state of health. To compare the appropriateness of each state of health feature, batteries were subjected to different discharge profiles and tested to failure. For each cycle, capacity, resistance, length of the constant current charge time and length of the constant voltage charge time were measured and compared based on their usefulness to estimate the state of health. Lastly, all the features were combined to give a fusion result for state of health estimation.
\end{abstract}

\section{INTRODUCTION}

Lithium-ion batteries have become an integral part of daily life. They are used in virtually all portable electronic devices and are becoming the battery of choice for hybrid and plug in electric vehicles. With the growing hybrid electric vehicle market, the use of batteries is expected to increase rapidly over the next few decades. Therefore, the reliability and maintenance associated with large scale battery usage must be managed. Battery management systems can assist in maintenance procedures by evaluating a battery's state of health ( $\mathrm{SOH})$. The $\mathrm{SOH}$ of a battery is a measure that describes how much the battery has degraded in health over the course of its life, and is often evaluated by the battery's internal resistance or its ability to deliver a given amount of charge. After a battery is assembled, it undergoes internal side reactions, which consume lithium and lead to gaseous and non-conductive byproducts that hinder the amount of electrical charge that can be stored by the battery. These reactions are accelerated by different usage and environmental conditions. Eventually, the charge storage capability (capacity) of the battery degrades below its required level of performance, to where it can no longer

Williard et al. This is an open-access article distributed under the terms of the Creative Commons Attribution 3.0 United States License, which permits unrestricted use, distribution, and reproduction in any medium, provided the original author and source are credited. perform its intended functions. Battery management systems must be able to predict when the batteries are approaching the threshold of remaining useful performance (RUP), so that users are provided adequate warning for battery replacement.

Traditionally, SOH and RUP have been evaluated with data measured during discharge. He (2011) used the discharge current integrated over time to calculate the maximum capacity during each charge/discharge cycle. The capacity decay with cycle number was modeled by a sum of exponentials equation, and then RUP measurements were predicted by extrapolating the model with a Bayesian Monte Carlo method. Andre (2013) incorporated SOH into a framework using a duel Kalman filter and support vector machine approach where $\mathrm{SOH}$ was updated with capacity and internal resistance measurements calculated by the change in voltage during pulsed discharge. Eddahech (2012) utilized equivalent series resistance from impedance spectroscopy measurements to determine $\mathrm{SOH}$ and predicted degradation behavior with recurrent neural networks. Other research Du (2010) \& Schmidt (2010) has described similar methods that utilize capacity and internal resistance as features to determine $\mathrm{SOH}$. However, influences such as temperature, vibration, and un-foreseen usage profiles give rise to uncertainties that are not addressed in these methods.

Rather than continue to increase the complexity and fidelity of SOH methods to improve accuracy, this paper minimizes factors that cause noise in $\mathrm{SOH}$ measurements by using multiple $\mathrm{SOH}$ features including ones taken from charging data. Unlike discharge, charge conditions are controlled and predictable. During charging, the same protocol is used throughout the entire life of the battery which eliminates uncertainties in the usage profile. Many charging circuits are designed so that the device's electronics are powered by the charger's power supply (rather than the battery). This allows battery charging to go undisturbed, even if the device is being used.

When determining $\mathrm{SOH}$ features, the method of charging utilized by a particular device should be considered. For this study, the conventional constant current constant voltage 
(CCCV) charging protocol will be assumed. The CCCV protocol can be easily implemented in practice by a number of commercially available integrated circuits such as Linear Technology's LT1512 or Texas Instrument's bq24157. The CCCV protocol uses a variable resistance to supply a constant current to the battery until a maximum threshold voltage is reached. Then, a constant voltage is applied to the battery until the current drops to a minimum threshold value. This charging method is preferred over constant voltage charging because by applying a constant voltage across the battery the current is allowed to float. This floating state leaves the battery vulnerable to high current charging which could reduce operational life and compromise safety. Instead CCCV applies a constant voltage charge at the end of the constant current charge as a "top off" to assure the battery has reached its maximum state of charge.

The $\mathrm{SOH}$ features extracted from the charging data were the length of the constant current charge time (CCCT) and the constant voltage charge time (CVCT). The availability of CCCT and CVCT as indicators for $\mathrm{SOH}$ was first suggested by Ramadass (2004) who modeled the changes in charging behavior due to capacity fade in lithium-ion batteries. The model predicted that increased resistance and decreased diffusion at the electrode/electrolyte interface decreases the rate at which lithium can be sufficiently intercalated into the anode during charging. Therefore, this model predicts that as the battery degrades, the CVCT will increase to accommodate the extra time needed for lithium-ions to diffuse into the graphite anode. Conversely, due to material degradation in the electrodes, the number of lithium insertion sites decrease with increased cycling. With less insertion sites, the time required for the cell to reach its maximum potential during charging decreases, suggesting that the CCCT will decrease as the battery undergoes more cycles.

The remainder of this paper describes the experimental procedure for performing lifecycle testing on three batteries. Then, $\mathrm{SOH}$ with respect to cycle number is evaluated using each of the four features (CCCT, CVCT, internal resistance, and capacity) for all of the batteries. Lastly, the features are systematically compared to determine their appropriateness for describing the true health status of the battery.

\section{EXPERIMENTAL METHOD}

Three prismatic 1.1Ah lithium-ion batteries were tested. $\mathrm{LiCoO}_{2}$ mixed with carbon as a conductive additive was used as the cathode, while layered graphite bound together with polyvinylidene fluoride was used as the anode. An electrolyte with equal parts ethylene carbonate (EC) and dimethyl carbonate (DMC), with $\mathrm{LiPF}_{6}$ as the lithium salt, provided the medium for ion transport between the two electrodes. The batteries underwent cycle life testing, where the same constant current constant voltage (CCCV) charging protocol was used for each battery during every charge cycle. Constant current charging was performed with a current of $0.55 \mathrm{~A}$ until the voltage of the battery reached $4.2 \mathrm{~V}$, and then the constant voltage phase was initiated, where the voltage was held at $4.2 \mathrm{~V}$ until the current fell to below $0.05 \mathrm{~A}$.

For each of the three batteries, a different discharge profile was used for cycle life testing Table 1. While the charge profile is the same for each battery, the particular discharge characteristics can have a residual effect on the following charge profile. To investigate how the method of discharge effects the following charge profile one of the batteries was programmed to discharge with varying current rates each cycle, while another battery was programmed to vary the depth of discharge each cycle. The first battery, to be denoted as battery $\mathbf{A}$ for the remainder of this paper, underwent a simple constant current discharge at $0.55 \mathrm{~A}$. The cut-off voltage denoting the end-of-discharge was set as $2.7 \mathrm{~V}$. The second battery, battery $\mathbf{B}$, underwent a constant current discharge, but the current rate was changed every cycle such that during the first cycle, the battery was discharged at a current of $0.11 \mathrm{~A}$; during the second cycle, $0.22 \mathrm{~A}$; during the third, $0.55 \mathrm{~A}$; during the fourth, $1.1 \mathrm{~A}$; during the fifth, $1.65 \mathrm{~A}$; and during the sixth cycle, the battery was discharged at $2.2 \mathrm{~A}$. This profile was repeated so that the battery was discharged at each current rate over every six cycle interval. For battery $\mathbf{C}$ the depth of discharge effect was evaluated by sporadically changing the cut-off voltage during discharge over the course of the cycle life. This caused the initial open circuit voltage at the beginning of the charging stage to be different for different cycles throughout the lifetime of the battery. Figure 1 shows the voltage profile of the battery over the course of 44 charge/discharge cycles. It can be seen that the cut-off voltage during discharge was periodically changed to simulate the user putting a battery on its charger before the battery has reached a fully depleted state.

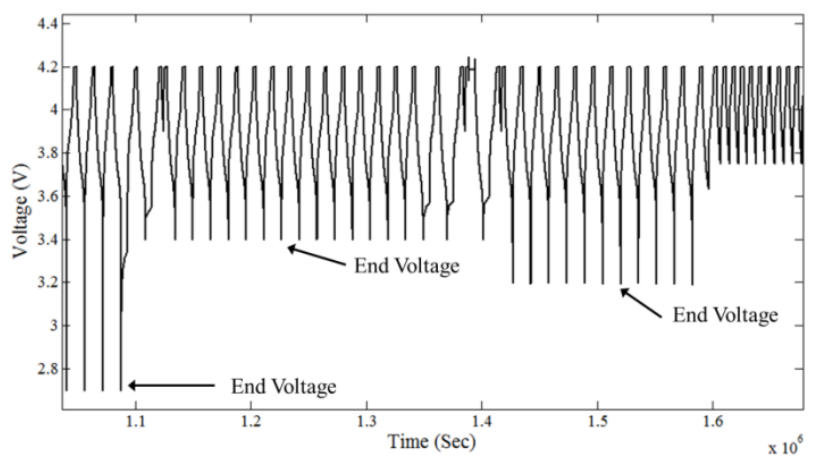

Figure 1 Various depth of discharge for battery $\mathrm{C}$ 


\begin{tabular}{|c|c|c|}
\hline Battery & Charge Profile & Discharge Profile \\
\hline A & $\mathrm{CCCV}$ & $\begin{array}{c}\text { Constant current discharge at } \\
0.55 \mathrm{~A} \text { from } 4.2 \mathrm{~V} \text { to } 2.7 \mathrm{~V}\end{array}$ \\
\hline B & $\mathrm{CCCV}$ & $\begin{array}{c}\text { Varying discharge current } \\
\text { alternating between } 0.11 \mathrm{~A}, \\
0.22 \mathrm{~A}, 0.55 \mathrm{~A}, 1.1 \mathrm{~A}, 1.65 \mathrm{~A}, 2.2 \mathrm{~A} \\
\text { each cycle from } 4.2 \mathrm{~V} \text { to } 2.7 \mathrm{~V}\end{array}$ \\
\hline $\mathrm{C}$ & $\mathrm{CCCV}$ & $\begin{array}{c}\text { Constant current discharge at } \\
0.55 \mathrm{~A} \text { varying cut off voltage } \\
\text { randomly between } 4.2 \mathrm{~V} \text { and } 2.7 \mathrm{~V}\end{array}$ \\
\hline
\end{tabular}

Table 1 Charge/discharge profiles

Figure 2 shows a typical current and voltage profile of the batteries for one charge/discharge cycle. It can be seen that a cycle is made up of three stages. During discharge, the current is denoted as a negative value and the voltage decreases non-linearly until it reaches a pre-designated cutoff voltage. During constant current charging, the current is held constant with a floating voltage until the voltage reaches its designated maximum charging voltage, at which point constant voltage charging begins. When the battery is held at a constant voltage, its current will decrease until it approaches a current that counter-acts the self-discharging current or until it reaches a predesigned cut-off value (as was the case in this test). The discharge capacity was found by integrating the discharge current by time; the resistance was found by applying a small current pulse at the end of charging, measuring the change in voltage and then dividing $d V$ by the magnitude of the current pulse; CCCT was found by measuring the length of time that the battery remained in the constant current phase of charging; and CVCT was found by measuring the length of time that the battery remained in the constant voltage phase of charging.

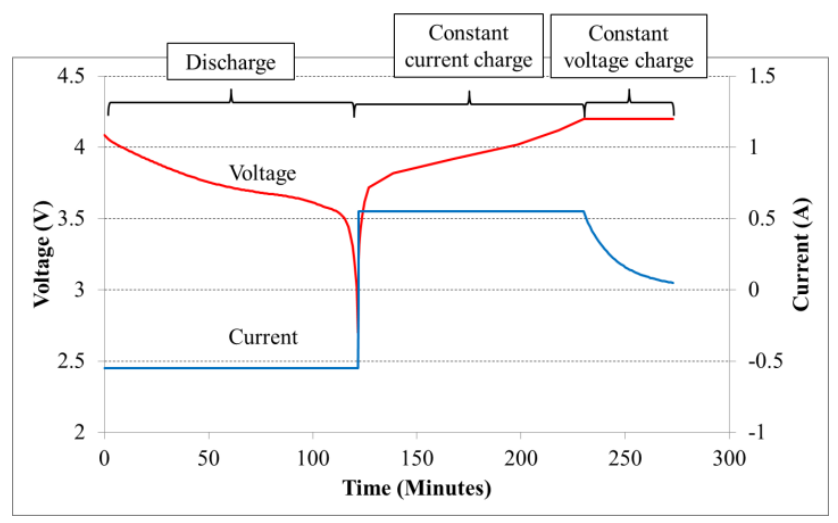

Figure 2 Charge-Discharge current and voltage profile

\section{RESULTS AND DISCUSSION}

Figure 3 shows each feature plotted versus cycle number for battery A. Because, this sample underwent constant current discharge at the same rate and the same depth of discharge for each cycle, it can be considered the simplest test case.
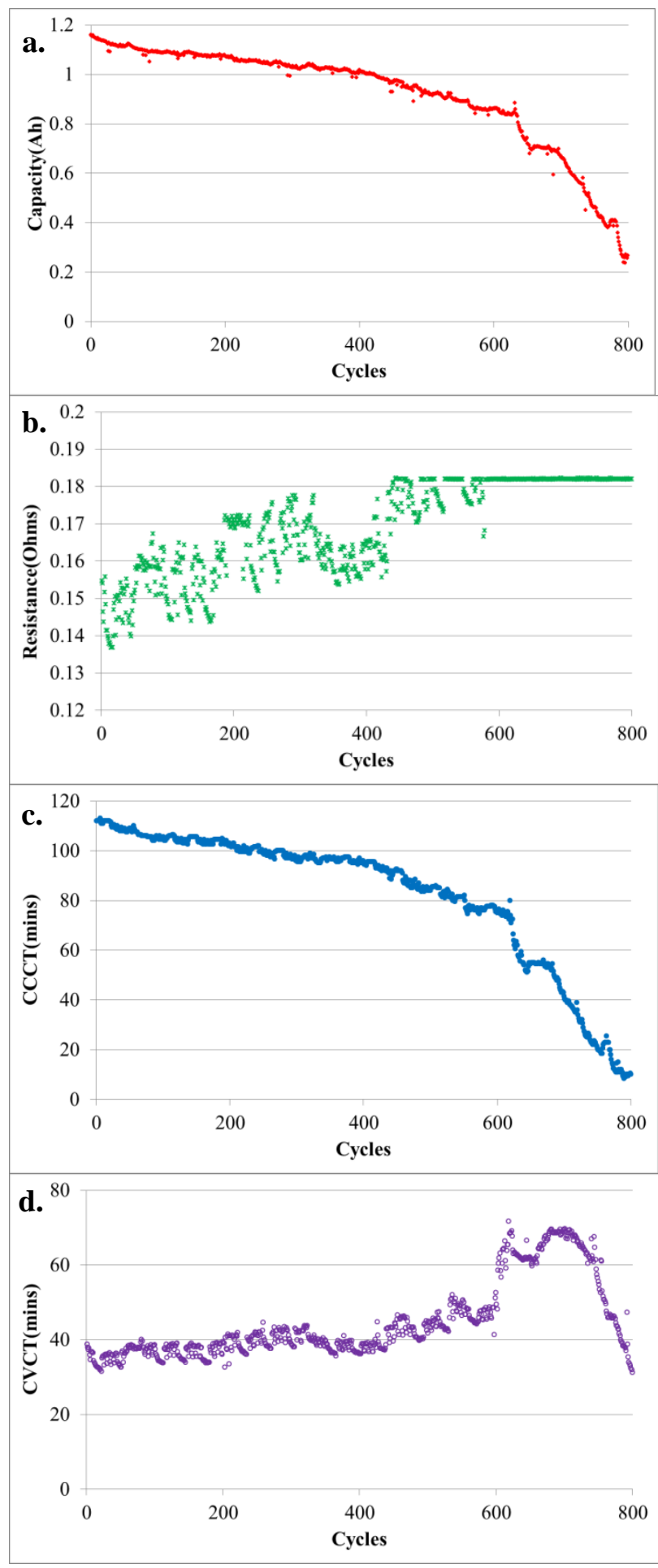

Figure 3 Each feature plotted for battery A, a. capacity b. resistance c. CCCT d. CVCT

Capacity and CCCT are nearly identical in form. Both features degrade in an exponential fashion as cycle number increases. Conversely, CVCT and resistance show a general increasing trend as cycle number increases. However, there were spans over the course of the battery's cycle life where 
resistance and CVCT displayed decreasing behavior. At around 700 cycles CVCT begins to drop sharply. Between cycles 300 and 400, resistance has a general decreasing behavior with increasing cycle number and after cycle 600 the resistance does not change with cycle number at all. Additionally, there is a substantial degree of noise that accompanies the CVCT and resistance measurements. These qualitative observations suggest that for the simple constant current, constant depth of discharge case, capacity and CCCT are more suitable indicators for $\mathrm{SOH}$.

Besides inherent noise and the consistency of feature trending, the length of time required to measure each feature can be used as a metric to evaluate the quality of a $\mathrm{SOH}$ feature. It is desirable that $\mathrm{SOH}$ measurements be performed quickly so that updates can be made in a reasonable time. An additional drawback of long $\mathrm{SOH}$ measurement times is that long measurements are susceptible to influencing factors such as dynamic discharge rates and temperatures. Battery A was discharged at half of its rated capacity; therefore the time required to measure the capacity is equivalent to twice the measured capacity during each cycle. Resistance can be measured almost instantly as $d V$ can be found with a short current pulse. At the beginning of life, discharge capacity requires the most amount of time to measure. However, as the battery loses the ability to store charge, capacity and CCCT require less time to be measured while the time required to measure CVCT increases.

Figure 4 shows the value of $\mathrm{SOH}$ calculated at each cycle from each of the four features for battery $\mathbf{A}$. The $\mathrm{SOH}$ in the features that decrease as cycle number increases (capacity and CCCT) were given by equation (1):

$$
S O H=\frac{F_{k}}{F_{\operatorname{avg}(1: 5)}}
$$

where $F$ is the given feature at a cycle number indicated by the subscript. Therefore at cycle $k$ the $\mathrm{SOH}$ is the value of the feature divided by the average of feature $F$ over the first five cycles. Averaging $F$ over the first five cycles gave a general value of $F$ that could be considered "healthy." Taking the averaging over the first five cycles reduces error that could be apparent from only using the first cycle as the healthy state. If more than 5 cycles are used, there is a risk that degraded data will be included into the healthy state. For CVCT and resistance which generally increase as cycle number increases, $\mathrm{SOH}$ was calculated by equation (2):

$$
S O H=\left(\frac{F_{k}}{F_{\operatorname{avg}(1: 5)}}\right)^{-1}
$$

Figure 5 shows each feature plotted for battery B which underwent discharge at 6 different current rates. It is clear that the discharge capacity and CCCT are dependent on the rate of discharge. Diffusion limited effects Du (2010) at the electrolyte/electrode interface prevent the full capacity from being utilized as the discharge current rate increases. In order to account for this dependency, a model relating capacity and CCCT to discharge rate would need to be incorporated into the $\mathrm{SOH}$ estimation procedure such as demonstrated in Schmidt (2010). However, model based $\mathrm{SOH}$ estimation requires recalibration over cycle life and between individual cells. As complexity in the discharge profile increases, the CVCT and resistance features become more appealing as they are not dependent on the specific discharge rate. At around 350 cycles, a peak in CVCT and resistance is observed. This peak could be indicative of specific internal degradation mechanisms that are not as apparent in the discharge capacity and CCCT. However, it is possible that this peak could trigger a false alarm as the premature rise in resistance and CVCT correspond to a reversible drop in $\mathrm{SOH}$ as shown in Figure 7.

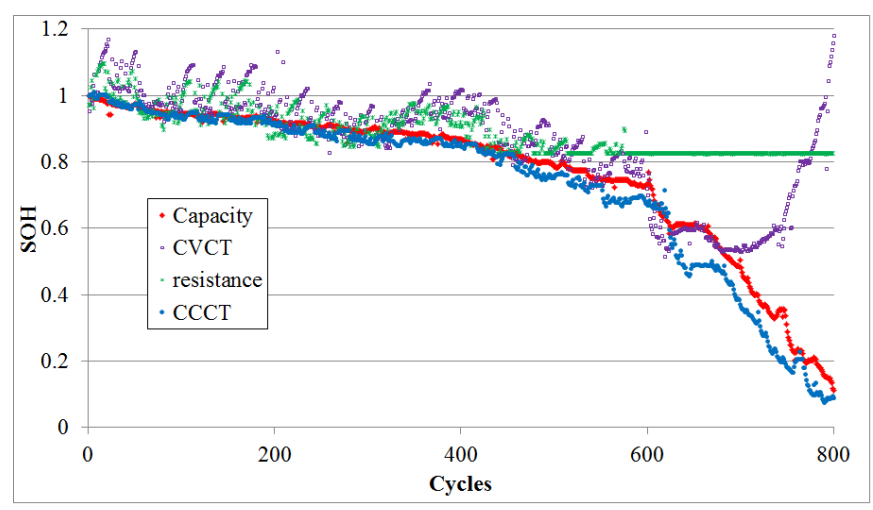

Figure $4 \mathrm{SOH}$ calculated from each feature for battery A

Figure 6 and Figure 8 show the features and corresponding $\mathrm{SOH}$ for battery $\mathrm{C}$ which was tested by keeping the discharge current constant at $0.55 \mathrm{~A}$ but sporadically changing the depth of discharge throughout cycle life. Again, capacity and CCCT show a heavy dependence on depth of discharge which would need to be accounted for by increased model complexity and constant recalibration. CVCT also appears to display a relationship with depth of discharge that changes as cycle number increases. Without compensation for depth of discharge, the $\mathrm{SOH}$ estimations for capacity, CVCT and CCCT are inaccurate. However, the resistance measurements for battery $\mathrm{C}$ are highly favorable, even more so than in the simplest test conditions shown in battery A. The resistance increases linearly throughout the entire cycle life with less noise than what was found in batteries A and B. However, because this linear trend in resistance increase is not consistently found in all batteries, relying solely on resistance can result in inaccurate $\mathrm{SOH}$ estimations. 

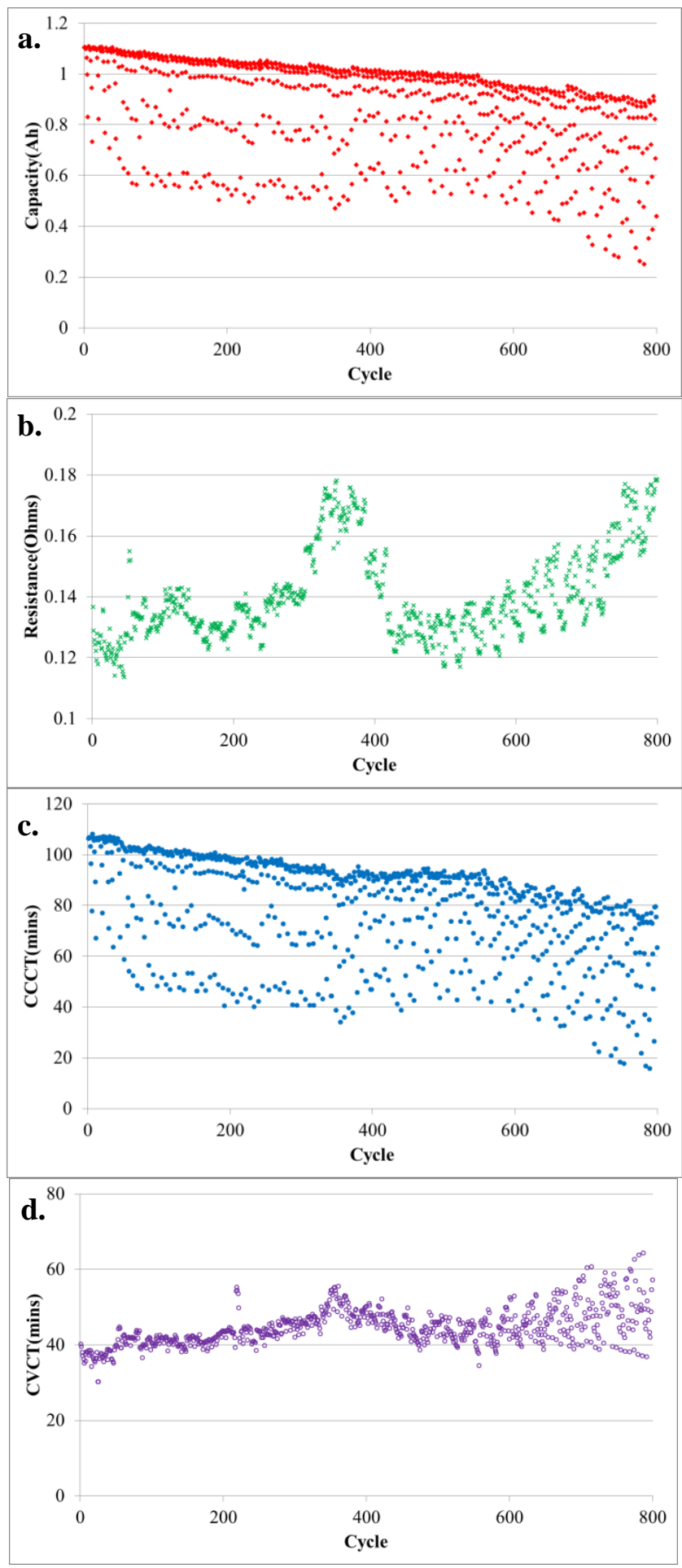

Figure 5 Each feature plotted for battery B, a. capacity b. resistance c. CCCT d. CVCT
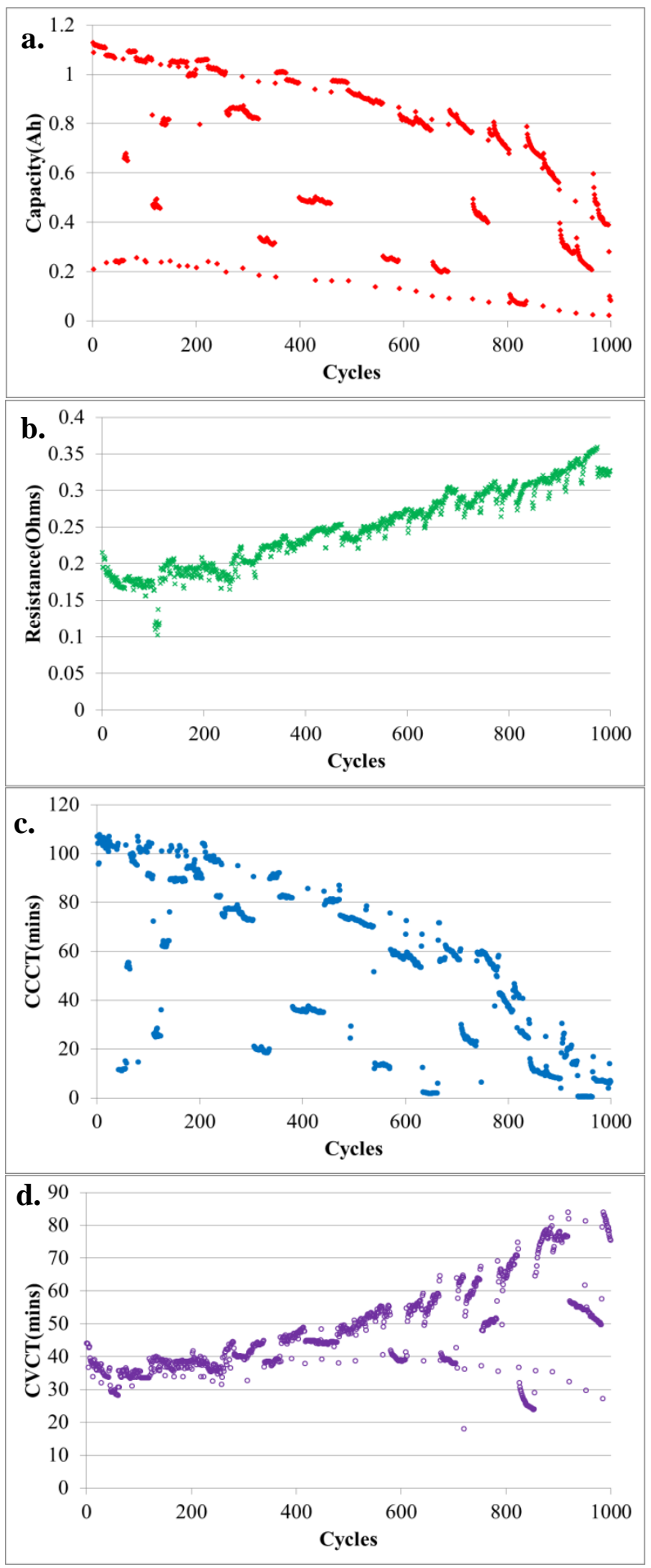

Figure 6 Each feature plotted for battery C, a. capacity b. resistance c. CCCT d. CVCT 


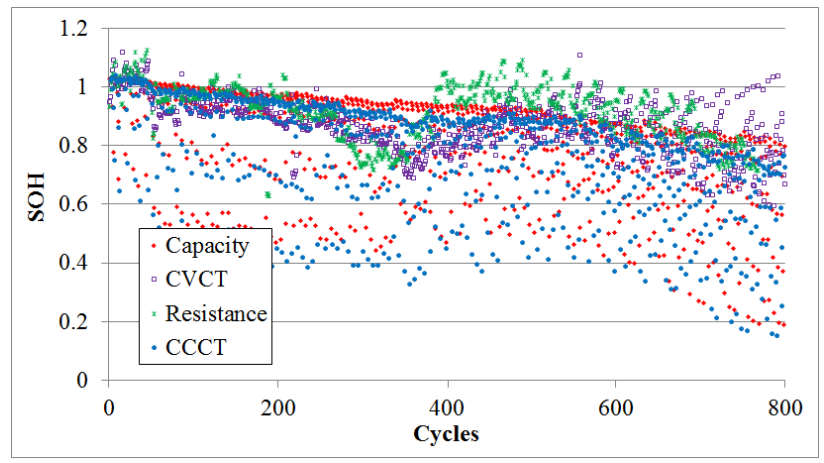

Figure $7 \mathrm{SOH}$ calculated from each feature for battery B

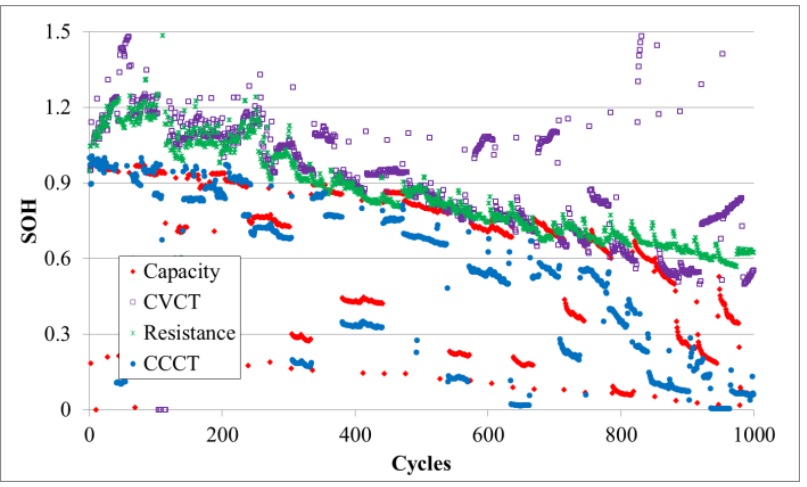

Figure $8 \mathrm{SOH}$ calculated from each feature for battery $\mathrm{C}$

\subsection{Fusion Results}

The results from comparing four different features at different cycle life conditions have indicated that there is no universal feature that can be used for accurate $\mathrm{SOH}$ estimation unless sufficient model adaptation is applied. However, each of the four features described can be extracted from battery data in a straight forward matter regardless of the complexity of the discharge conditions. Rather than construct a new model for each battery which would require sufficient cycle life testing and parameter initialization, the four features described in this paper can instead be combined through a generalized fusion method.

To perform data fusion each $\mathrm{SOH}$ feature $\mathrm{F}_{i}^{c}$ described by Eq.(1) and Eq.(2) where $i$ indicates each of the 4 features described in this paper and $c$ indicates the cycle number, is combined using a weighted average. During each cycle $c$, the distribution of the $\mathrm{SOH}$ features is characterized by the beta function. The beta function is chosen because it has the same range as $\mathrm{SOH}(0 \rightarrow 1)$, it is flexible for characterizing a wide range of probability distribution functions, and it is a conjugate prior probability distribution allowing for easy Bayesian updating. Therefore, $\mathrm{SOH}$ is characterized at each cycle $c$ by:

$$
f_{c}(S O H ; \alpha, \beta)=\frac{S O H^{\alpha-1}(1-S O H)^{\beta-1}}{B(\alpha, \beta)}
$$

In order to determine the parameters $\alpha$ and $\beta$, the mean of the beta function is set equal to the weighted mean of the observed features:

$$
\frac{\alpha}{\alpha+\beta}=\frac{\sum_{i=1}^{4} w_{i} \mathrm{~F}_{i}}{\sum_{i=1}^{4} w_{i}}
$$

where $w_{i}$ corresponds to the weight of each feature. For each cycle the values of $\alpha$ and $\beta$ are determined from Eq.(3):

$$
\begin{gathered}
\alpha_{c}=\sum_{i=1}^{4} w_{i}^{c} F_{i}^{c} \\
\beta_{c}=\sum_{i=1}^{4} w_{i}^{c}\left(1-\mathrm{F}_{i}^{c}\right)
\end{gathered}
$$

After the distribution of $\mathrm{SOH}$ is determined for a particular cycle, the point value of $\mathrm{SOH}$ for a given cycle number is found by taking the maximum likelihood of $f_{c}(\mathrm{SOH} ; \alpha, \beta)$ :

$$
S O H_{c}=\operatorname{argmax}\left(f_{c}(S O H ; \alpha, \beta)\right)
$$

After each cycle the weights are updated based on the following equation:

$$
w_{i}^{c+1}=w_{i}^{c}+\left(1-\left|S O H_{c}-\mathrm{F}_{i}^{c}\right|\right)
$$

Final smoothing of the SOH metric was performed with a 10 cycle window moving average. Figure 9 shows the results of parameter fusion for battery $\mathrm{A}, \mathrm{B}$ and $\mathrm{C}$. By fusing all four of the parameters, the $\mathrm{SOH}$ with cycle relationship becomes more linear and less noisy than any one of the parameters by itself. The exception of this is in the case of Battery $\mathrm{C}$ in which resistance proved to be an accurate representation of $\mathrm{SOH}$. However, because resistance is not a reliable metric across all batteries, there is a clear advantage in using parameter fusion for $\mathrm{SOH}$ estimation. Out of all the test parameters, the cut-off voltage had the most influence on the $\mathrm{SOH}$ metric as seen by the variability in battery $C$. This can be accounted for by increasing the window of the moving average.

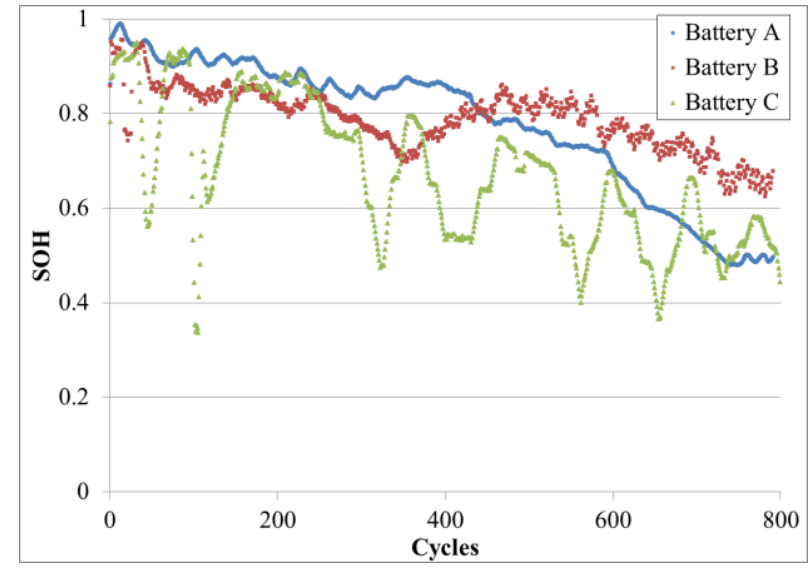

Figure 9 Fusion of the four parameters using the median 


\section{Conclusions}

Capacity and resistance are typically the only features used for determining $\mathrm{SOH}$ in lithium-ion batteries. However, these features do not accommodate for the dependency on user profiles and environmental conditions. Typical approaches to $\mathrm{SOH}$ estimation introduce adaption schemes which require a deep understanding of input to output relationships and Gaussian noise parameters. Additionally, many researchers fail to acknowledge the inconsistency in the trend of resistance increase with battery degradation. Rather than continue to increase model fidelity and enhanced model adaption for state estimation, this paper demonstrates the effectiveness of increasing the number of features extracted from battery. By considering data taken during the charging profile of the battery, it was found that the length of time the battery spent in each of the constant current and constant voltage phases of charging correlated to state of health. To create a single metric of state of health the two features taken from the charging data were fused with resistance and discharge capacity. Using all four features the distribution of $\mathrm{SOH}$ was characterized by equating the weighted mean of the features to the mean of the Beta distribution. The fused $\mathrm{SOH}$ measure was found by taking the maximum likelihood of the resulting distribution and then updating each of the weights.

\section{REFERENCES}

1. Andre D, Appel C, Guth T, Sauer D, Advanced Mathematical Methods of SOC and SOH estimation for lithium-ion batteries. Journal of Power Sources 224 (2013) 20-27
2. Du W, Gupta A, Zhang X, Sastry A, Shyy W, Effect of Cycling Rate, Particle Size and Transport Properties on Lithium-Ion Cathode Performance, International Journal of Heat and Mass Transfer 53 (2010) 3552$3561 \mathrm{~A}$.

3. Eddahech A, Briat O, Bertrand N, Deletage J, Vinassa $\mathrm{J}$, Behavior and State of Health Monitoring of Lithium Ion Batteries Using Impedance Spectroscopy and Recurring Neural Networks. Electrical Power and Energy Systems 42 (2012) 487-494

4. He W, Williard N, Osterman M, Pecht M, Prognostics of Lithium-Ion Batteries Based on Dempster-Shafer Theory and The Bayesian Monte Carlo Method. Journal of Power Sources 196 (2011) 10314- 10321

5. Ramadass P, Haran B, Gomadam P, White R, Popov B, Development of first principles capacity fade model for Li-ion cells Journal of the Electrochemical Society. 2004, Volume 151. Issue 2. Pages A196-A203

6. Schmidt, Bitzer M, Imre A, Guzzella L, Model-Based Distinction and Quantification of Capacity Loss and Rate Capability Fade in Li-ion Batteries Journal of Power Sources 195 (2010) 7634-7638

\section{ACKNOWLEDGMENT}

This material is based upon work supported by the National Science Foundation under grant number (\#1234451). The authors would also like to thank L-3 Communications for their fellowship granted to the lead author along with the Center for Advanced Life Cycle Engineering (CALCE) at the University of Maryland and the more than 100 companies and organizations that support its research annually. 